Meta

Journal des traducteurs

Translators' Journal

\title{
Gestion et organisation de la documentation au Bureau des traductions du Canada
}

\section{Suzanne Richer}

Volume 28, numéro 2, juin 1983

URI : https://id.erudit.org/iderudit/003949ar

DOI : https://doi.org/10.7202/003949ar

Aller au sommaire du numéro

Éditeur(s)

Les Presses de l'Université de Montréal

ISSN

0026-0452 (imprimé)

1492-1421 (numérique)

Découvrir la revue

Citer cette note

Richer, S. (1983). Gestion et organisation de la documentation au Bureau des traductions du Canada. Meta, 28(2), 217-220. https://doi.org/10.7202/003949ar d'utilisation que vous pouvez consulter en ligne.

https://apropos.erudit.org/fr/usagers/politique-dutilisation/ 


\section{GESTION ET ORGANISATION DE LA DOCUMENTATION AU BUREAU DES TRADUCTIONS DU CANADA.*}

Suzanne Richer

Aborder ou encore accepter de discuter de documentation avec un groupe de traducteurs et de terminologues représente tout un défi pour un bibliothécaire. Je vous propose donc de traiter des services documentaires du double point de vue de leur organisation et de leur gestion en fonction d'une clientèle hautement spécialisée et non pas du point de vue de la typologie des services professionnels.

Parler de documentation avec des traducteurs et des terminologues, c'est aussi s'immiscer dans leur intimité professionnelle. Tous, vous avez développé vos méthodes de recherche bibliographique et de consultation documentaire; tous, vous vous tenez au courant des nouvelles parutions dans le domaine de l'édition que ce soient des dictionnaires, des encyclopédies, des ouvrages généraux ou spécialisés de sorte que vous êtes autant à la page que bien des bibliothécaires.

Par contre, le bibliothécaire vous étonne et vous déçoit aussi par son acharnement à organiser « votre» documentation, c'est-à-dire le fonds bibliographique dont dispose votre employeur. Pourquoi, vous demandez-vous, le bibliothécaire s'obstine-t-il à déplacer les collections sur les étagères, à développer des règlements concernant le prêt entre bibliothèques ou les heures d'ouverture des salles de consultation et de lecture?

Il serait certes facile et expéditif de répondre que le bibliothécaire est à la recherche de modalités et de dénominateurs communs lui permettant de mettre à la disposition d'une majorité, des ressources bibliographiques souvent très limitées. Les terminologues nous répètent qu'ils doivent tout consulter et tout examiner pour conduire à terme une recherche thématique valable. Comment non seulement le

* Colloque de Glendon, 1980. 
bibliothécaire, mais l'organisme ou l'agence pour lesquels travaillent ces spécialistes peuvent-ils tout obtenir soit par achat ou encore par prêt? Comment?

Il faut donc attaquer le problème à la base en gérant la documentation et les collections; on doit définir des politiques, des procédures et des règlements. Il faut aussi simultanément gérer les besoins des clientèles par des mécanismes tels que l'établissement de profils documentaires. L'expérience que l'on vit actuellement au Bureau des traductions est valable évidemment pour ce milieu de travail et ne peut être transplantée intégralement ailleurs sans y apporter au préalable des changements et des modifications tenant compte des utilisateurs, des besoins documentaires et des ressources disponibles. Au Bureau, toute la philosophie du service documentaire repose sur la réalité «information/usager/bibliothécaire». Au départ, il est important sinon essentiel de faire comprendre aux usagers la distinction entre «information» et «documentation», définition que l'on pourrait naïvement résumer ainsi : information $=$ contenu et documentation ou documents au sens large $=$ contenant. La communication est souvent fondée sur ces définitions, car le bibliothécaire tentera de transmettre à son client l'information plutôt que de le renvoyer continuellement et automatiquement à des ouvrages. Le bibliothécaire se veut, au tout début du dialogue, l'agent de dépouillement et de dissémination de l'information.

Quel est donc le fondement de la gestion documentaire au Bureau des traductions et sur quoi repose-t-elle? L'expérience de gestion documentaire est basée sur un plan quinquennal établi en 1978 et dont voici les principales lignes directrices :

Ce plan-cadre déterminait les besoins actuels de la clientèle, les ressources humaines, financières et documentaires dont la Direction de la documentation disposait ef proposait finalement un programme pour les années 1978-1983. Les ressources humaines nécessaires aux réalisations professionnelles et techniques ont été identifiées, les modalités et les activités du service de référence et du traitement de la documentation ont été analysées et la structure du réseau de bibliothèques et de modules documentaires a été arrêtée. Le modèle documentaire choisi est plutôt conventionnel puisqu'il est basé sur un ensemble de petites bibliothèques locales (bibliothèques de sections de traduction et de terminologie) appuyé par 4 bibliothèques dites «de réseau» appelées à compléter les collections des bibliothèques de section et à dispenser des services de référence ponctuelle et thématique, de prêts entre bibliothèques, de choix de collection. Cet ensemble de modules documentaires est voué à la communication avec l'usager, à la diffusion de l'information et à l'analyse constante des besoins locaux.
La Direction de la documentation est une composante de la Direction générale de la terminologie et de la documentation (DGTD) et doit donc tenir compte du mandat général de la DGTD. Par exemple, dans le document $« L a$ Banque de terminologie du gouvernement canadien; aperçu général $\gg$ on y introduit la possibilité éventuelle d'une liaison «base de données documentaires» et «base de données terminologiques» en vue de constituer une banque d'informations terminologiques intégrée permettant aux traducteurs et aux autres clients de la Banque d'accéder à des informations terminologiques et documentaires structurées.

La Direction de la documentation (DD) a donc comme mandat général de fournir aux traducteurs, terminologues et interprètes du $\mathrm{Bu}$ reau des traductions, le soutien documentaire nécessaire à l'exécution de leurs travaux. La structure administrative de la DD repose sur l'interrelation de ses trois divisions (voir organigramme annexé); chacune a son plan de travail axé sur la gestion documentaire des ressources globales telles que déterminées par les hauts fonctionnaires du ministère.

Regardons brièvement l'organisation des trois divisions et analysons, en cours de description, leurs outils de gestion.

\section{La Division des services publics}

Son outil de gestion, c'est le profil d'intérêt qui est un inventaire des besoins des différentes clientèles établi conjointement par le bibliothécaire et l'usager. $\dot{A}$ quoi sert ce profil? Toute la philosophie de la Politique de développement et de rationalisation des collections est régi par ces profils d'intérêt. Les sections de traduction et de terminologie sont toutes profilées afin de déterminer les besoins en documentation des composantes opérationnelles du Bureau; les descripteurs employés pour bâtir le profil allient les sujets et la nomenclature de la typologie des documents pour donner une image exacte des besoins actuels de la section profilée. Un système de mise à jour des profils est décrit afin de conserver des informations courantes sur les besoins déterminés. Cet outil est complété par un Index des profils qui apporte des réponses immédiates aux questions suivantes: Quels sont les sujets des principales collections? Quelles sections développent plus particulièrement des collections dans telle ou telle discipline.

Les profils d'intérêt déterminent aussi le degré d'importance des collections par l'indication d'un paramètre qualifiant le besoin documentaire; les demandes de documents peuvent donc être «prioritaires», « essentielles » ou «utiles $»$; donc, toutes les demandes de documents sont complétées selon les principes de la Politique de développement et de rationalisation des collections et on peut conclure que les 
collections du Bureau sont développées en fonction des besoins réels. L'élément de rationalisation des collections est de fait le contrôle de l'outil de gestion. Ce monitoring des collections est assuré par le bibliothécaire qui amène sa clientèle à juger de la pertinence de telle ou telle collection dans tel ou tel lieu géographique. Doit-on acheter en double exemplaire des ouvrages coûteux, volumineux et peu consultés? Le client des services documentaires prend conscience à ce moment des facteurs économiques de la documentation: le prix, mais aussi les coûts de traitement et d'entreposage. Il doit choisir: tout avoir dans sa section ou accepter de partager pour acquérir une plus grande variété de titres. L'usager du Bureau apprend donc à se déplacer (et ce dans des limites raisonnables) et il découvre ainsi de nouveaux bassins documentaires dans sa périphérie de travail.

La demande de document remplie par un client est analysée par le bibliothécaire; il détermine l'action à prendre. Doit-il acheter ou emprunter l'ouvrage demandé ? Se reportant aux profils d'intérêt, le bibliothécaire est en mesure de décider au meilleur des intérêts de ses clients.

La Politique de développement et de rationalisation des collections détermine aussi les ouvrages dits de "La trousse des traducteurs et des terminologues $»$. Cette liste a été élaborée par les deux parties en cause; la DD maintient un inventaire des trousses et peut ainsi s'assurer que les traducteurs et les terminologues ont les outils indispensables à leur travail. On peut prévoir combien d'exemplaires des nouvelles éditions on doit acheter (par exemple, le nouveau Harrap's qui vient de paraître).

En plus du profil d'intérêt pour le développement des collections et par conséquent l'identification des besoins des clients, la Division des services publics utilise aussi «le profil documentaire " pour établir des bibliographies courantes et rétrospectives soit manuellement ou par ordinateur. Encore une fois, le profil permet de répondre rapidement aux demandes. Les projets spéciaux en terminologie thématique sont profilés et ces profils et ces bibliographies établis peuvent servir à d'autres demandes sur le même sujet. On peut combiner des profils pour établir des bibliographies spécifiques, comparatives, etc.

\section{La Division des services techniques}

Les demandes d'acquisition de matériel documentaire conformes aux profils d'intérêt sont effectuées et les ouvrages sont ensuite traités. La section des acquisitions répond à plus de 7000 demandes de documents (année 1979) en provenance de quelque 140 unités de travail du Bureau réparties à travers le Canada et traite les 1000 abonnements à plus de 720 titres de périodiques. Je passe sous silence les contrôles administratifs normaux et réguliers qu incombent à tout service d'achat. Le traitement effectué par la section de catalogage touche la classification, le catalogage et le codage de source. La DD utilise le système LC (Library of Congress) avec vedettes-matières en anglais et en français. Elle se sert des services informatiques d'UTLAS (University of Toronto Library Automation Systems).

La mandat de cette division est d'assurer le développement et le maintien du catalogue collectif du Bureau. Encore un outil de gestion des collections. Le catalogue collectif DOTT ( $D O$ cumentation pour la Traduction et la Terminologie; catalogue collectif de la documentation au Bureau des traductions) offert sur support micrographique (COM) est diffusé à toutes les sections de traduction et permet aux utilisateurs de 1) localiser la documentation et de la consulter sur place ou encore de l'emprunter 2) dresser des bibliographies cursives à même le fonds documentaire du Bureau.

\section{La Division du réseau de bibliothèques}

Son rôle est d'offrir localement et donc à tous les traducteurs et terminologues des services documentaires professionnels par le biais des grandes bibliothèques du réseau et des différentes bibliothèques de section. Cette division est vouée à la communication entre la DD et ses usagers et vice-versa. Elle supporte les programmes de la DD et propose des modalités de services. Elle assure le respect des politiques de la DD et analyse l'impact de ces politiques sur le service documentaire. Par le biais des comités de bibliothèque, la communication verticale s'établit. Ses outils de gestion sont très «ordinaires»; le maintien de statistiques de fréquentation des bibliothèques et d'utilisation des services spécialisés permet de profiler chaque bibliothèque.

Les trois divisions sont sous la direction de bibliothécaires professionnels responsables des opérations techniques et professionnelles de leur secteur d'activité, de la coordination de leurs activités avec les autres divisions et finalement de la gestion des ressources humaines qui leur sont allouées.

En terminant, je désire toucher rapidement au rôle de la Direction de la documentation dans les secteurs nationaux et internationaux de la bibliothéconomie et des sciences de l'information. La DD agit à titre de conseiller auprès de certaines agences de traduction désireuses de se doter de services documentaires structurés. Des échanges d'experts et de bases de données documentaires sont aussi inscrits au programme de la DD; des ententes ont été réalisées avec l'Office de la langue française, le Conseil international de la langue française (CILF), le Bureau de terminologie de la Commission des communautés européennes de 
Bruxelles et de Luxembourg. La DD est évidemment très intéressée aux projets et aux réalisations qui touchent le secteur de la documentation.

Au tout début de ce texte, je vous ai dit que je ne traiterais pas de la documentation du point de vue «services». Je ne peux vous laisser sans vous donner an moins la liste des produits documentaires que la DD offre à ses usagers :

1. DOTT (DOcumentation pour la $T$ raduction et la Terminologie; catalogue collectif de la documentation au Bureau des traductions)

2. LISTE DES PUBLICATIONS EN SERRIE (répertoire des titres reçus avec mention des codes de localisation)

3. DOCUMENTATION (ouvrages récemment catalogués et dossiers assemblés par la Direction de la documentation)

4. INDEX DES PROFILS D'INTÉRÊT

5. LISTE DES PUBLICATIONS DISPONIBLES

6. DIC/VOC (des bibliographies)

7. DIC/DOTT : liste par sujet des dictionnaires, encyclopédies, glossaires et vocabulaires répertoriés dans DOTT.

À la Direction de la documentation du Bureau des traductions, les bibliothèques du réseau sont le fondement de la relation «information/usager tandis que le catalogue collectif en est l'instrument qui permet la diffusion de l'information stockée. L'équipe de communication formée de bibliothécaires et de bibliotechniciens n'a pas comme seule tâche la diffusion de l'information et de la documentation mais doit aussi générer de nouvelles formules documentaires, établir des mécanismes de dépistage des besoins des usagers et aussi créer des besoins chez l'usager afin de maintenir le dynamisme évolutif des services documentaires. 